\title{
Vaginal foreign body mimicking cervical cancer in postmenopausal woman - case study
}

\author{
Michał Ciebiera, Aneta Słabuszewska-Jóźwiak, Witold Ledowicz, Grzegorz Jakiel \\ First Department of Gynaecology and Obstetrics, The Medical Centre of Postgraduate Education, Warsaw, Poland
}

\begin{abstract}
We present a case report of a 73-year-old, postmenopausal woman with detailed history of breast cancer and oncology treatment including tamoxifen therapy. She presented at the clinic of gynecology and obstetrics with recurrent inflammation of the urinary and genital tract and suspicion of a cervical mass. She also presented occasional abdominal complaints and malodorous vaginal discharge. These symptoms were observed in the patient for several years. Before hospitalization she received many kinds of empirical, antimicrobial treatment such as chlorquinaldol, metronidazole, nifuratel, and nystatin. She did not receive further guidance from doctors about the causes of ailments and further diagnostic and treatment capabilities. In our clinic a detailed diagnostic process including ultrasound transvaginal examination and a minisurgical procedure revealed the presence of a vaginal foreign body (which turned out to be a plastic, shampoo bottle cap) surrounded by a mass of inflamed tissue mimicking a cervical tumor. All symptoms and complaints subsided after surgical removal of the foreign body and antibacterial therapy with metronidazole and cefuroxime. Our study draws attention to the need of thorough gynecological care including prophylaxis, especially in the case of complaints of an intimate nature. Even trivial, frequently occurring disorders can be dangerous and require proper and responsible doctor's supervision and management through the healing process.
\end{abstract}

Key words: foreign body, vagina, inflammation, infection, ultrasonography.

\section{Introduction}

Vaginal foreign bodies are a relatively unusual occurrence in gynecology. They are most often found in young girls under the age of 4 , whereas in women of reproductive age and their postmenopausal peers they are detected sporadically. In children, foreign bodies are usually inserted out of curiosity while the infants explore their bodies, but they may also be a sign of sexual abuse with the use of various objects. In adults, foreign bodies are most frequently inserted for sexual stimulation during intercourse or masturbation, although cases of patients attempting to correct pelvic organ prolapse with foreign bodies have also been documented. The range of possible vaginal foreign bodies is wide, including fruit and vegetables, batteries, small metal objects, tools, toys, as well as erotic gadgets.

The aim of our study is to present a case of a postmenopausal woman with recurrent urinary and genital tract infections secondary to the presence of a vaginal foreign body.

We report a rare pathology of a persistent vaginal foreign body in a postmenopausal woman. The medical history of the patient, documented with photos and images, as well as recent literature reports on the topic, constitute the material for this case study.

\section{Case study}

A 73-year-old woman was admitted to a gynecological ward due to chronic inflammation of the urinary and genital tract, accompanied by occasional abdominal complaints and malodorous vaginal discharge. The patient had had an earlier diagnosis of a tuberous lesion in the region of the cervix and posterior vaginal fornix, confirmed on ultrasound, but the diagnostic process was discontinued due to unknown causes. The woman, during tamoxifen treatment at the time of the study, had undergone oncologic treatment two years previously: unilateral (left-sided) mastectomy due to breast cancer, followed by adjuvant therapy. The patient denied any treatments due to other diseases; she was receiving magnesium and potassium supplementation.

In the course of the last 2 years, the patient was treated at an out-patient gynecological clinic due to recurrent urinary and genital tract infections. Abdominal pain of moderate intensity was reported. There was 
no history of urinary tract, rectal, or defecation-related complaints. Due to vaginal inflammation, the patient had received a wide spectrum on antifungal agents, including chlorquinaldol, metronidazole, nifuratel, nystatin, and others. Pre-admission vaginal culture revealed numerous colonies of Staphylococcus aureus. The gynecologist had prescribed oral clindamycin. Pap-smear revealed cervicitis, with no signs of cervical intraepithelial neoplasia. One year previously, a biopsy of the posterior vaginal fornix and the region with chronic inflammatory changes had been performed.

On admission, the patient was in good overall condition, afebrile, with no abdominal complaints. Gynecological examination revealed a movable, anteflexed uterine cervix of normal size. A solid, tuberous mass, tender on palpation, was found in the region of the posterior vaginal fornix. No palpable adnexal pathologies were detected. Vaginal discharge was intensely malodorous. The patient did not consent to speculum or transvaginal ultrasound examinations. Transrectal ultrasound test revealed an anteflexed uterine cervix of non-homogeneous echogenicity $(63 \times 58 \mathrm{~mm}$ in size $)$. The endometrium and the ovaries were not visualized by ultrasound. No free fluid was found in the Douglas pouch. A solid, nonhomogeneous, vascularization-rich mass $(32 \times 18 \mathrm{~mm}$ in size) was detected at the vaginal vault.

Laboratory results were: WBC $7.12 \mathrm{~K} / \mu \mathrm{l}$; RBC $4.55 \mathrm{M} /$ $\mu \mathrm{l} ;$ HGB $14.5 \mathrm{~g} / \mathrm{dl}$; PLT $254 \mathrm{~K} / \mu \mathrm{l}$; CRP $17.2 \mathrm{mg} / \mathrm{l}$. The consulting team analyzed patient data, symptoms and the

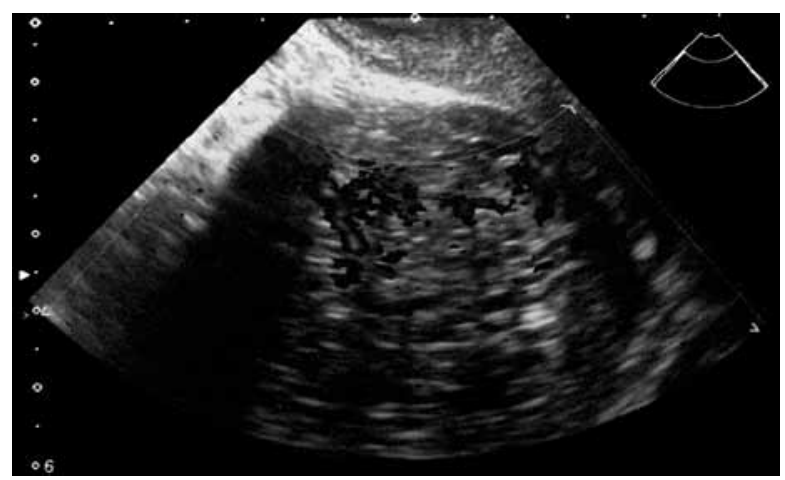

Fig. 1. Rich vascularization of the lesion visualized by power Doppler

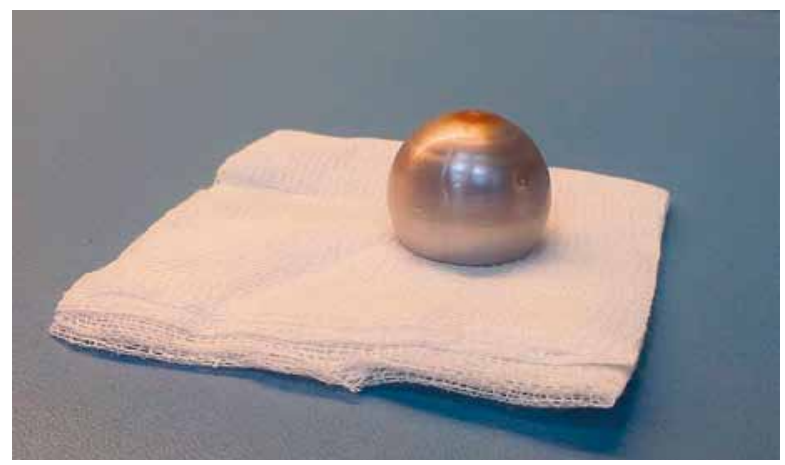

Fig. 3. Foreign body - shampoo bottle cap obtained results, due to ambiguous signs and findings, and deemed the patient eligible for surgical removal of the vaginal lesion.

The patient received short-acting general anesthesia through an i.v. and, after speculum insertion, the suspicious lesion was visualized at the vaginal vault. A hard, shiny, smooth surface was revealed upon incision. After evacuation, a foreign body, which turned out to be a plastic shampoo bottle cap, was enucleated from the mass. The area of the original location of the foreign body was left open, without signs of bleeding. No injury or damage to the vaginal walls and the adjacent organs, including the rectum, and no signs of rectovaginal fistulas were found.

After the procedure, the patient received preventive metronidazole with cefuroxime. Culture results were positive for Klebsiella pneumoniae, which was nonresistant to the administered drugs. Vaginal douching with povidone-iodine and octenidine solution was performed on subsequent days. The patient was discharged in good overall condition. The follow-up at 1 and 2 months showed a significantly subsiding inflammation and undisturbed healing. The patient was lost to follow-up some time later.

\section{Discussion}

Vaginal foreign bodies constitute a specific problem in gynecological practice. They may be inserted into the

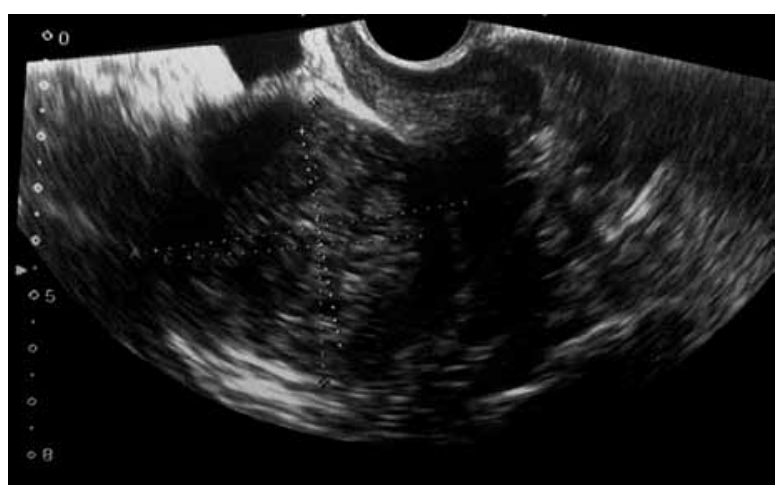

Fig. 2. Heterogeneous mass in the cervical area

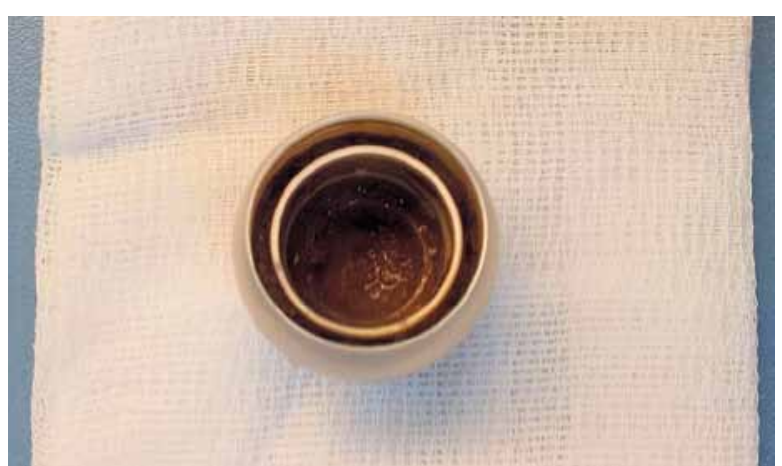

Fig. 4. Foreign body - shampoo bottle cap, different angle 
vagina due to various reasons, for example during menstrual bleeding, sexual activity, masturbation, or rape [1-5]. Cases when foreign bodies are used as a kind of pessary by elderly patients with pelvic organ prolapse have also been reported $[6,7]$. Exploration and experimentation with one's own body are most commonly observed in childhood or in patients with mental disturbances [8-11]. Various objects have been reported to be found in the vagina, including fruit and vegetables, batteries, toys, sexual accessories, construction tools, and even polyurethane foam [5, 12-14].

Complaints caused by foreign bodies depend on their kind, chemical composition, structure, shape, and time elapsed since insertion in the vagina. The affected individuals usually present with abdominal pain, intensified discharge, spotting, bleeding, dyspareunia, and foul odor from the genital and urinary tract. Chronic presence of a foreign body in the vagina results in tissue pressure and changes, as the foreign body hurts, perforates and causes inflammation of the affected region. Ulceration of the surrounding tissues, peritonitis, and migration of the foreign body to other cavities have been reported as well. Often, the foreign body may cause vesicovaginal and rectovaginal fistulas [4, 15-17]. Single cases of neoplastic processes due to the presence of a foreign body have also been observed [7, 18, 19]. In the case of our patient, the woman denied sexual intercourse and masturbation, and her husband had died many years previously. The patient insisted the plastic cap must have entered the vagina accidently, for example during a bath. A more detailed history could not be obtained due to obvious embarrassment and discomfort of the woman. Also, she avoided more detailed questions by claiming she had difficulty recalling past events. Non-specific ultrasound findings, oncologic history, and tamoxifen use must have alerted the doctors with regard to the etiology of the lesion. The coincidence between the prevalence of changes within the reproductive organ and therapy with selective estrogen receptor modulators, e.g. tamoxifen in breast cancer patients, has been reported. Ultrasound differentiation of such changes is a complex and challenging task, as many images are ambiguous and their interpretation by various specialists may also vary [20-24].

The above-described case offers a chance to emphasize the great importance of regular and complex gynecological check-up in postmenopausal women, often combined with additional tests, e.g. transvaginal ultrasound, especially in cases with oncologic history. Gynecologists ought to be patient and vigilant when collecting medical history, as elderly women often suffer from other diseases, ignore gynecologic complaints, forget about them, feel embarrassed, or mistakenly associate some pathologies with their advanced age.

\section{Disclosure}

Authors report no conflict of interest.

\section{References}

1. Jaluvka V, Novak A. Vaginal foreign bodies in women in postmenopause and in senium. Eur J Obstet Gynecol Reprod Biol 1995; 61: 167-169.

2. Chapman GW. An unusual intravaginal foreign body. J Natl Med Assoc 1984; 76: 811-812.

3. Herbenick D, Reece M, Sanders SA, et al. Prevalence and characteristics of vibrator use by women in the United States: results from a nationally representative study. J Sex Med 2009; 6: 1857-1866.

4. Puppo A, Naselli A, Centurioni MG. Vesicovaginal fistula caused by a vaginal foreign body in a 72-year-old woman: case report and literature review. Int Urogynecol J Pelvic Floor Dysfunct 2009; 20: 1387-1389.

5. Ogbonmwan SE, Varghese S, Phleming A, et al. Unusual presentation of vaginal foreign bodies. J Obstet Gynaecol 2005; 25: 322-323.

6. Dasari P, Sagili H. Incarcerated foreign body in the vagina - a metal bangle used as a pessary. BMJ Case Reports 2012; 2012.

7. Malhotra N, Chanana C, Lal S. Forgotten vaginal pessary discovered after 30 years. J Gyn Obset 2007; 6: 13.

8. Paradise JE, Willis ED. Probability of vaginal foreign body in girls with genital complaints. Am J Dis Child 1985; 139: 472-476.

9. Stricker T, Navratil F, Sennhauser FH. Vaginal foreign bodies. J Paediatr Child Health 2004; 40: 205-207.

10. Herman-Giddens ME. Vaginal foreign bodies and child sexual abuse. Arch Pediatr Adolesc Med 1994; 148: 195-200.

11. Krasucki C, Kemp R, David A. A case study of female genital self-mutilation in schizophrenia. Br J Med Psychol 1995; 68: 179-186.

12. Rogowski A, Wodzislawska A, Doniec J, et al. Foreign body in vagina polyurethane foam. Case report. Ginekol Pol 2010; 81: 232-234.

13. Melamed Y, Dalyahu Y, Vaiman R, et al. Foreign objects in the vagina of a mentally ill woman: case series. Gen Hosp Psychiatry 2007; 29: 270-272.

14. Ahmidat A, Tailor A. Unusual presentation of vaginal foreign body. J Obstet Gynaecol 2010; 30: 873-875.

15. Binstock MA, Semrad N, Dubow L, et al. Combined vesicovaginal-ureterovaginal fistulas associated with a vaginal foreign body. Obstet Gynecol 1990; 76: 918-921.

16. Pokorny SF. Long term intra-vaginal presence of foreign body in children. A preliminary study. J Reproductive Med 1994; 39: 931-935.

17. Deligeoroglou E, Deliveliotou A, Laggari V, et al. Vaginal foreign body in childhood: a multidisciplinary approach. J Paediatr Child Health 2006; 42: 649-651.

18. Nwosu EC, Rao S, Igweike C, et al. Foreign objects of long duration in the adult vagina. J Obstet Gynaecol 2005; 25: 737-739.

19. Jain A, Majoko F, Freites O. How innocent is the vaginal pessary? Two cases of vaginal cancer associated with pessary use. J Obstet Gynaecol 2006; 26: 829-830.

20. Kalampokas T, Sofoudis C, Anastasopoulos C, et al. Effect of tamoxifen on postmenopausal endometrium. Eur J Gynaecol Oncol 2013; 34: 325 328.

21. Bezircioglu I, Baloglu A, Tarhan MO, et al. Evaluation of endometrium by transvaginal ultrasonography and Doppler in tamoxifen-treated women with breast cancer. Eur J Gynaecol Oncol 2012; 33: 295-299.

22. Munro MG; Southern California Permanente Medical Group's Abnormal Uterine Bleeding Working Group. Investigation of women with postmenopausal uterine bleeding: clinical practice recommendations. Perm J 2014; 18: 55-70.

23. Le Donne M, Alibrandi A, Ciancimino L. Endometrial pathology in breast cancer patients: Effect of different treatments on ultrasonographic, hysteroscopic and histological findings. Oncol Lett 2013; 5: 1305-1310.

24. Polin SA, Ascher SM. The effect of tamoxifen on the genital tract. Cancer Imaging 2008; 8: 135-145. 